\title{
To Circ or Not to Circ: Clinical and Pharmacoeconomic Outcomes of a Prospective Trial of Topical Steroid versus Primary Circumcision
}

\author{
Yuri D. Nobre, Ricardo G. Freitas, Maria J. Felizardo, Valdemar Ortiz, Antonio Macedo Jr.
}

Section of Pediatric Urology, Department of Urology, Federal University of Sao Paulo, Sao Paulo, SP, Brazil

\begin{abstract}
Purpose: To compare the efficacy and costs of circumcision versus topical treatment using a prospective pharmacoeconomic protocol.

Materials and Methods: We treated 59 patients (3-10 years of age) randomized into two groups: 29 underwent an 8-week course of topical treatment with $0.2 \%$ betamethasone-hyaluronidase cream twice a day; and 30 underwent circumcision. Topical treatment success was defined as complete exposure of the glans. In cases of treatment failure, circumcision was performed and its cost imputed to that of the initial treatment. The pharmacoeconomic aspects were defined according to the Brazilian National Public Health System database and the Brazilian Community Pharmacies Index.

Results: The two groups were statistically similar for all clinical parameters evaluated. Topical treatment resulted in complete exposure of the glans in $52 \%$ of the patients. Topical treatment was associated with preputial pain and hyperemia. However, treatment suspension was unnecessary. Minor complications were observed in $16.6 \%$ of the surgical group patients. The mean cost per patient was US\$ 53.70 and US\$ 125.20, respectively, for topical steroid treatment (including the costs related to treatment failure) and circumcision. The total costs were US\$2,825.32 and US\$ 3,885.73 for topical treatment and circumcision, respectively.

Conclusions: Topical treatment of phimosis can reduce costs by $27.3 \%$ in comparison with circumcision. Therefore, topical treatment of phimosis should be considered prior to the decision to perform surgery.
\end{abstract}

Key words: phimosis; circumcision; male; steroids; pharmacoeconomics

Int Braz J Urol. 2010; 36: 75-85

\section{INTRODUCTION}

Circumcision is considered the elective treatment for phimosis in newborn infants and children. In the United States, circumcision is the fourth most common surgical procedure, performed in $65.3 \%$ of the male population (1).

Various factors, such as social norms, cultural traditions and religious beliefs, influence the decision of whether or not to perform circumcision. The American Academy of Pediatrics, in one of their most recent publications on this subject, stated that there is no absolute medical indication for the routine circumcision of newborn infants, and that, despite the potential medical benefits and advantages, circumcision also presents disadvantages and risks. When truly indicated, the risks and benefits should be discussed with parents, who should be asked to give written informed consent (2). 
In the $1990 \mathrm{~s}$, due to the controversy surrounding the topic of when circumcision is indicated, various studies were carried out in order to investigate alternative treatments for phimosis. Consistent success rates were achieved with the use of topical corticosteroids $(3,4)$.

Pharmacoeconomic studies help identify, calculate and compare the costs and risks of specific programs or therapies, as well as their benefits. Such studies also help determine which alternatives provide the best results and constitute the most practical use of the resources invested. Therefore, pharmacoeconomics is a relevant tool in the decision-making process, introducing the concept of economic rationality into health care systems in order to complement clinical decision making (5). There have been few studies on the economic impact of circumcision versus topical treatment of phimosis. Therefore, we conducted a prospective randomized study comparing, from the pharmacoeconomic point of view, the implications of topical treatment versus those of circumcision in cases of phimosis.

\section{MATERIALS AND METHODS}

A prospective randomized study was carried out in order to evaluate the clinical response to topical treatment of pathologic phimosis, comparing it to that of circumcision.

We included 85 children, from 3 to 10 years of age, diagnosed with phimosis. We defined as phimosis, in this age bracket, phimosis type I (no retraction of the foreskin) and type II (external urethral meatus exposure only), in accordance with the classification system devised by Kayaba et al. (6).

The study was previously approved by the Ethics Committee on Research of our University, and all parents or guardians were instructed and signed the written informed consent before starting the study.

In the clinical group, 42 patients were treated with two daily applications of $0.2 \%$ betamethasone and hyaluronidase cream for 8 weeks. The same physician examined all of the patients. Prior to the beginning of treatment and in every subsequent consultation, patients were photographed.
The therapeutic response was considered favorable only if the glans was easily and completely exposed during the subsequent physical examination, without a phimotic ring or balanopreputial adhesions (Kayaba type V). The therapeutic response was considered unfavorable if there was no exposure of the glans or there was only partial exposure of the glans due to balanopreputial adhesions or a phimotic ring. Therefore, the criterion adopted in order to define treatment success was the same as the expected outcome of circumcision: complete exposure of the glans.

Patients who responded well to the topical treatment underwent outpatient follow-up evaluations at 2 and 4 months after the end of treatment.

Patients who did not respond to the topical treatment by week 8 or who presented recurrence during the follow-up period underwent circumcision.

Patients in the surgical group were evaluated and then referred to undergo circumcision. Patients underwent outpatient follow-up evaluations on postoperative days 15,30 and 120 .

All surgical procedures were carried out at our institution. The anesthesia protocol was general inhalation anesthesia with sevoflurane, in conjunction with nerve block of the penis.

In the present study, the pharmacoeconomic analysis was of the cost-minimization type. Only the costs of the surgical procedure and clinical treatment (those directly related to the health care system: medical care, medications and medical materials) were taken into consideration. Indirect costs related to lost productivity, as well as intangible costs (those related to pain, suffering and impaired quality of life), were not calculated.

The pharmacoeconomic analysis was carried out from the perspective of a Public Health Hospital, and the resources included in the cost analysis were identified through communications with officials of the Brazilian United Health Care System (BUHCS). The medication costs that were not available in the public health system database were determined by consulting the Brazilian Community Pharmacies Index.

For patients in the surgical group, the costs were calculated separately for each of the following aspects: medical visits; anesthetic medications; 
surgical materials; medical and nursing team; and medications for the treatment of adverse effects.

For patients in the clinical group, the costs of the medical visits were calculated separately from those of the medications. Since some of the patients in the clinical group were eventually referred to surgery, those costs were incorporated into the final costs for that group (Figure-1).

Statistical evaluation of data was carried out by means of the chi-square test, Fisher's exact test, likelihood ratio test and Student's t-test. The level of significance was set at $p \leq 0.05$. For the pharmacoeconomic evaluation of the groups, we used the nonparametric Mann-Whitney test, for which the level of significance was also set at $\mathrm{p} \leq 0.05$.

\section{RESULTS}

Of the 85 children included in the study, 43 were allocated to the surgical group and 42 were al- located to the clinical group. From the surgical group, 13 children were excluded: 1 due to uncooperativeness; 2 due to refusal to undergo surgery; and 10 due to failure to return for surgery. Coincidentally, 13 children were also excluded from the clinical group: 1 due to irregular use of medication; 1 due to being clinically diagnosed with balanitis xerotica obliterans; 1 due to previous use of medication; and 10 due to failure to appear for medical visits. The mean age of the population in the study was 5.81 years.

All clinical parameters evaluated were statistically similar after the comparison between the groups (Table-1).

In the surgical group, the mean surgical time was $34.5 \mathrm{~min}$, the mean time to emergence from anesthesia was $43 \mathrm{~min}$, and the mean postoperative stay in the infirmary was $47.3 \mathrm{~min}$. On postoperative day 15, 5 patients presented complications of the procedure: 2 due to infection caused by improper cleaning; and 3 due to hematomas and scarring on the glans. These complications were considered mild and were treated

success

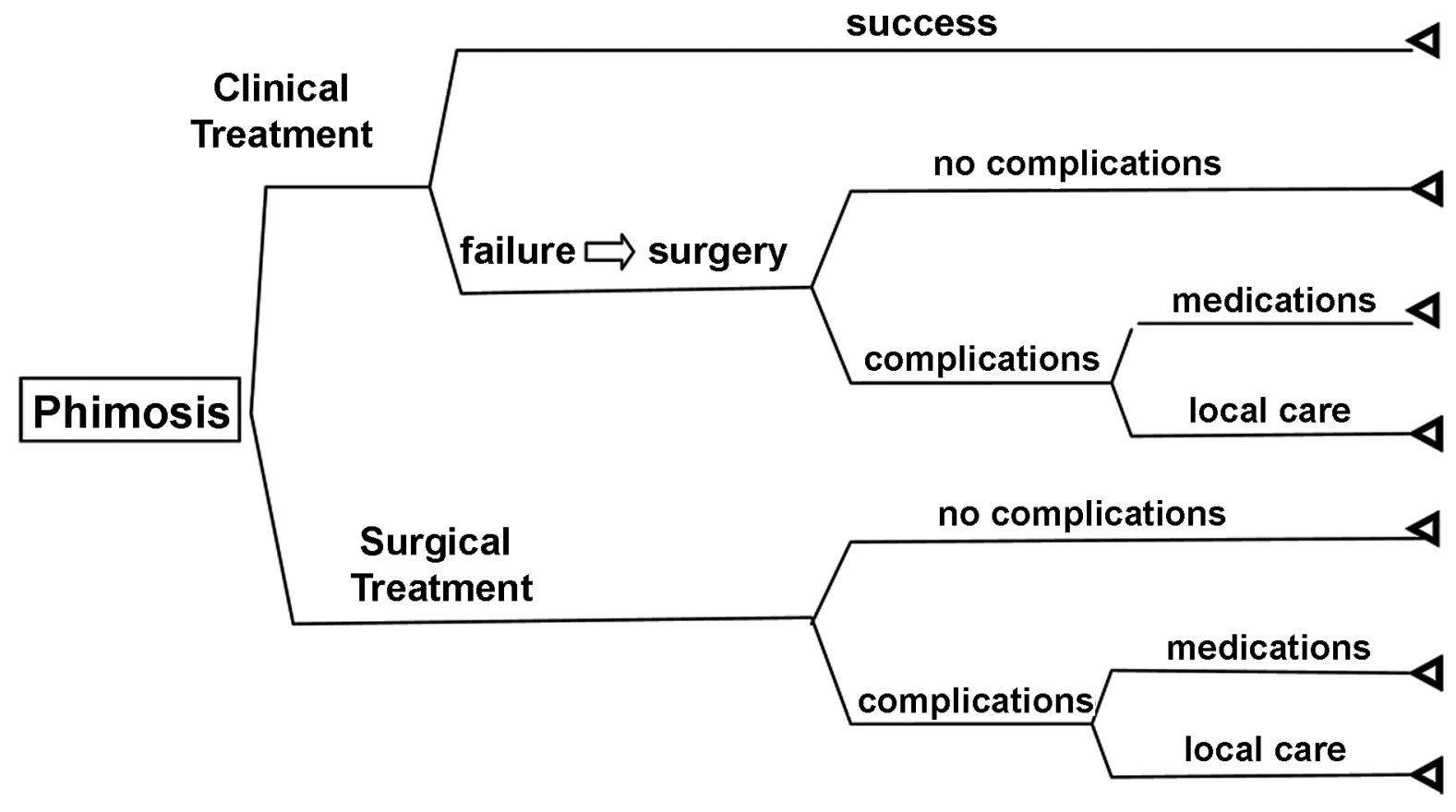

Figure 1-Flowchart of the pharmacoeconomic study design. 
Table 1 - Distribution of clinical findings regarding the incidence of balanitis, exposure of the urethral meatus and excess foreskin.

\begin{tabular}{|c|c|c|c|c|c|c|}
\hline Variable & & Surgical & & Clinical & p Value & Test \\
\hline Balanitis & & & & & 0.9 & Chi-square test \\
\hline No & 22 & $73.3 \%$ & 21 & $72.4 \%$ & & \\
\hline Yes & 8 & $26.7 \%$ & 8 & $27.6 \%$ & & \\
\hline Variable & & Surgical & & Clinical & p Value & Test \\
\hline Visible meatus & & & & & 0.205 & Chi-square test \\
\hline No & 13 & $43.3 \%$ & 19 & $65.5 \%$ & & \\
\hline Yes & 17 & $56.7 \%$ & 10 & $34.5 \%$ & & \\
\hline Variable & & Surgical & & Clinical & p Value & Test \\
\hline Excess foreskin & & & & & 1.000 & Fisher's exact test \\
\hline No & 3 & $10 \%$ & 2 & $7 \%$ & & \\
\hline Yes & 27 & $90 \%$ & 27 & $93 \%$ & & \\
\hline
\end{tabular}

with intensive local care. In the subsequent follow-up visits, all of the patients presented penile conditions that were considered normal, with proper formation of scar tissue.

In the clinical group, $15(51.7 \%)$ of the 29 patients presented complete exposure of the glans at 2 months after the end of treatment. The topical treatment resulted in adverse effects or complications in 10 patients: 4 patients experienced hyperemia and a burning sensation in the foreskin; 4 patients experienced a burning sensation in the foreskin; and 2 patients presented balanopreputial hyperemia. No intervention or treatment interruption was necessary in any of the cases.

In the clinical group, patients in whom treatment success was achieved were monitored for 4 months. Neither loss of exposure of the glans nor recurrence was detected.

Clinical treatment failure occurred in 14 cases, and those patients were referred to circumcision. Of those 14 patients, 2 failed to return for surgery. For the 12 who underwent surgery, the mean surgical time was $31.2 \mathrm{~min}$, the mean time to emergence from anesthesia was $30 \mathrm{~min}$, and the mean postoperative stay in the infirmary was $60 \mathrm{~min}$.
Of the 12 clinical group patients undergoing circumcision, 4 presented postoperative complications: 1 due to infection caused by improper cleaning, which demanded oral antibiotic treatment and local care; 1 due to bleeding and scars on the gland, which was treated locally; 1 due to pyogenic granuloma; and 1 due to an inflammatory process close to urethral meatus. All of the clinical group patients presented resolution of the complications during the follow-up period.

\section{Pharmacoeconomic Analysis}

In the pharmacoeconomic analysis, we evaluated the direct costs related to treatment of the two groups. It is of note that, as previously mentioned, the costs related to circumcision of patients in the clinical group were included in the final costs for that group (Figures 2-3).

In the pharmacoeconomic evaluation, the costs were higher in the clinical group only in terms of the medical visits and the use of topical medication. Costs regarding medical and nursing staff, anesthetic medications and surgical materials were higher in the 


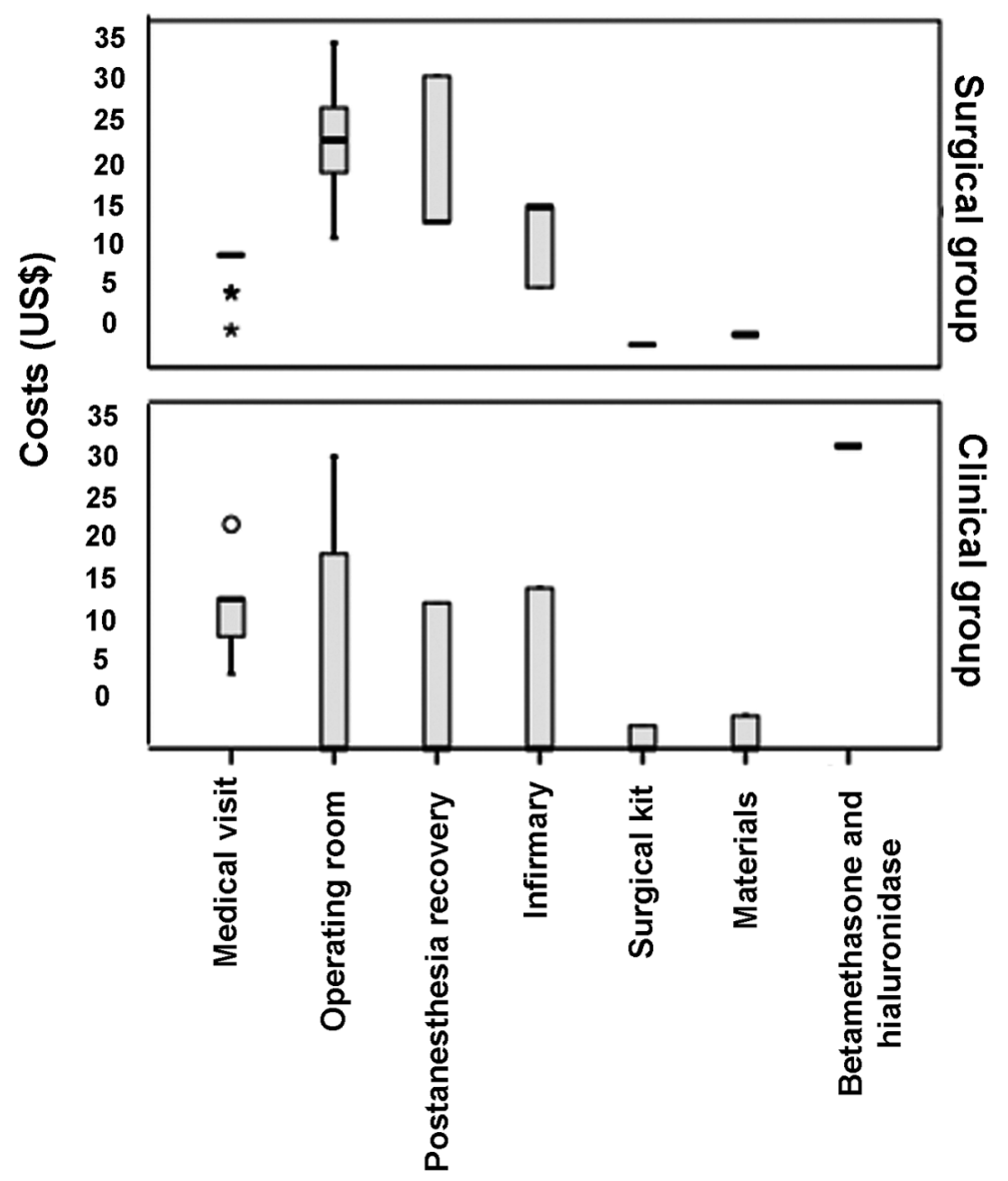

Figure 2-Box plot of the cost analysis of the parameters investigated in both groups.

surgical group than in the clinical group $(\mathrm{p}<0.001)$. No statistical difference was found in costs regarding medications used for the treatment of postoperative complications.

As can be seen in Figure-4, the analysis of the total costs per group, revealed greater economy in the clinical group than in the surgical group US\$ $2,825.32$ vs. US $\$ 3,885.73 ; \mathrm{p}=0.068$; median, US\$ 53.70 vs. US\$ 125.20 .

Comparing the median cost to the set price stipulated by the BUHCS through the Authorized Hospital Admissions System (US\$ 52.20) for every patient who underwent circumcision, we found considerable discrepancy between the amount paid by the BUHCS and the actual hospital costs of the circumcisions (Figure-5).

\section{COMMENTS}

Circumcision is classically considered the gold standard for the treatment of phimosis. Approximately one-sixth of males worldwide are circumcised (7). The indications for performing neonatal circumcision are controversial. Prominent among the factors favoring neonatal circumcision are the lower incidence of penile cancer, the lower incidence of balanitis/urinary infections, and the prevention of sexually transmitted diseases, including AIDS $(8,9)$. In addition, the incidence of postoperative complications is low. These benefits are sufficiently relevant to guarantee the routine practice of neonatal circumcision (8). However, most studies in which routine circumcision is recommended are retrospective studies, 


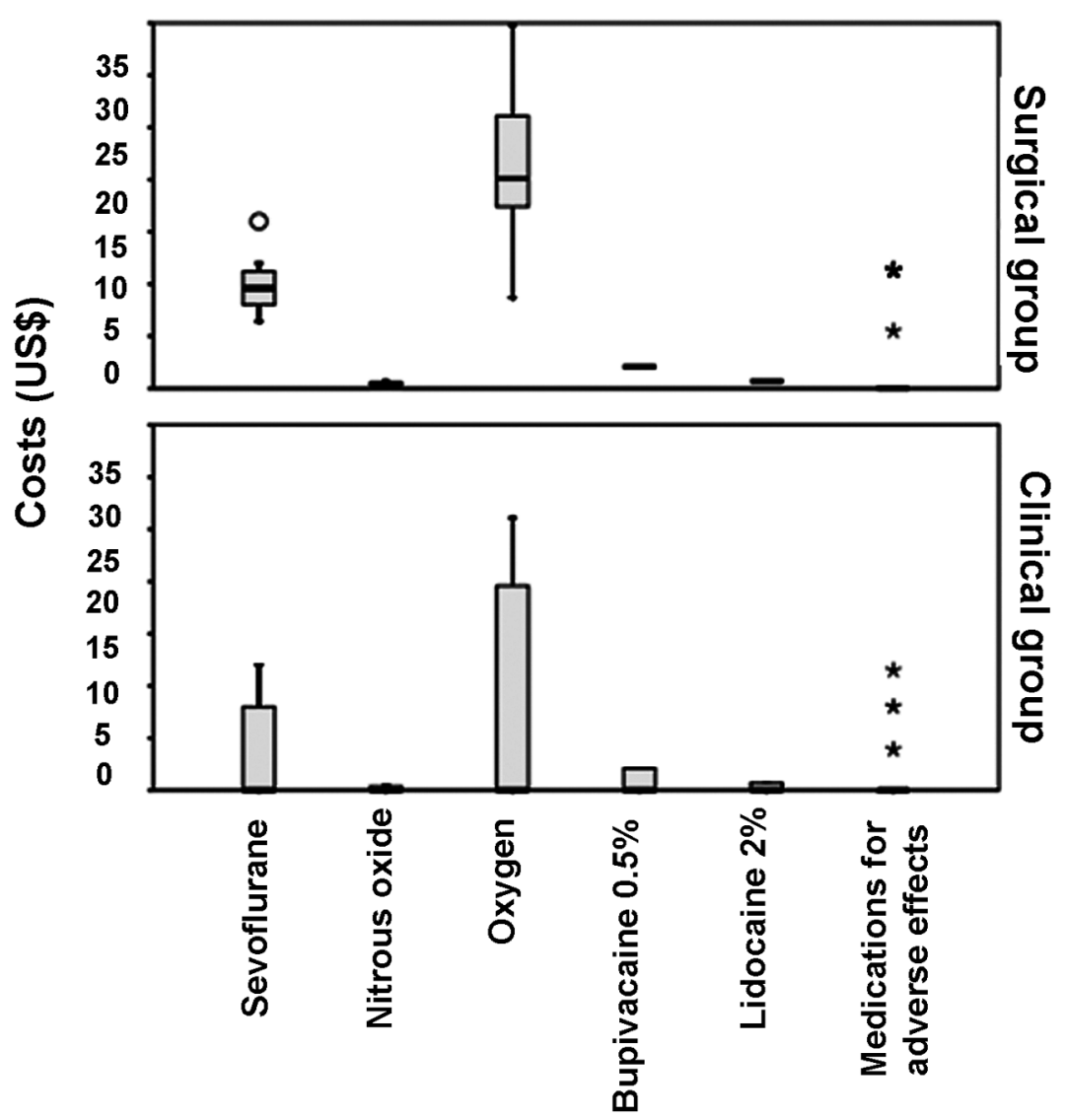

Figure 3 - Box plot of the cost analysis of the parameters investigated in both groups.

and the validity of the conclusions should be critically investigated. Circumcision is, arguably, a preventive health care measure, which, in theory, facilitates hygiene. However, the potential benefits occur years after the surgery, and few studies involve adequate, timely follow-up evaluation (10).

In Brazil, we do not adopt the concept of neonatal circumcision and most indications for surgery are considered when the child is about to leave diapers and only patients with phimotic ring are candidates for this kind of treatment.

The clinical treatment of phimosis consists of the gentle retraction of the foreskin and application of a topical corticosteroid to the foreskin and phimotic ring, with the objective of achieving complete exposure of the glans. The effects of topical corticosteroids, which have anti-inflammatory and immunosuppressive properties, on the metabolism of arachidonic acid and inhibition of interleukin-1 synthesis have been well established. Topical corticosteroids can have an antiproliferative effect on the dermal matrix, decreasing skin thickness and obliterating the stratum corneum $(11,12)$.

Hyaluronidase acts by modifying intercellular permeability and reducing tissue resistance, thereby increasing the diffusion of substances between planes (13).

In a pioneer study on the application of topical corticosteroid in boys with phimosis who were candidates for circumcision, clinical treatment was shown to be viable and efficacious (3). Topical treatment of phimosis with corticosteroids has characteristics that make it quite attractive as the first approach to the treatment of phimosis. The method is safe, and complication rates are similar to those found for circumcision. In addition, corticosteroid treatment presents high cure rates and low costs, as well as be- 


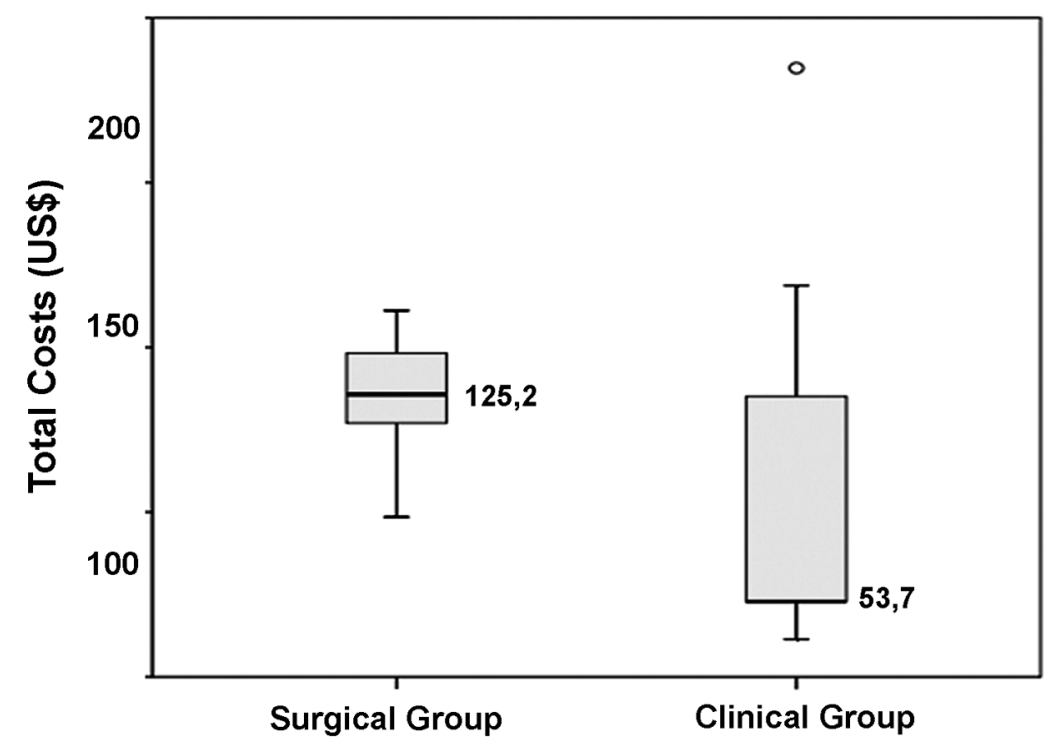

Figure 4-Box plot of the analysis of total costs in both groups.

ing easily administered and being well accepted by family members (14-16).

In the present study, $51.7 \%$ of the patients in the clinical group presented complete exposure of the glans, and none presented recurrence or lack of exposure of the glans during the 4 months of posttreatment follow-up evaluation. It is of note that we adopted a strict criterion for treatment success, excluding partial exposure of the glans, and that, in $65.5 \%$ of the patients, only the urethral meatus was exposed (Kayaba type I) prior to treatment. In multivariate analyses, Kayaba type I presentation has been described as a determining factor for corticosteroid treatment success (16).

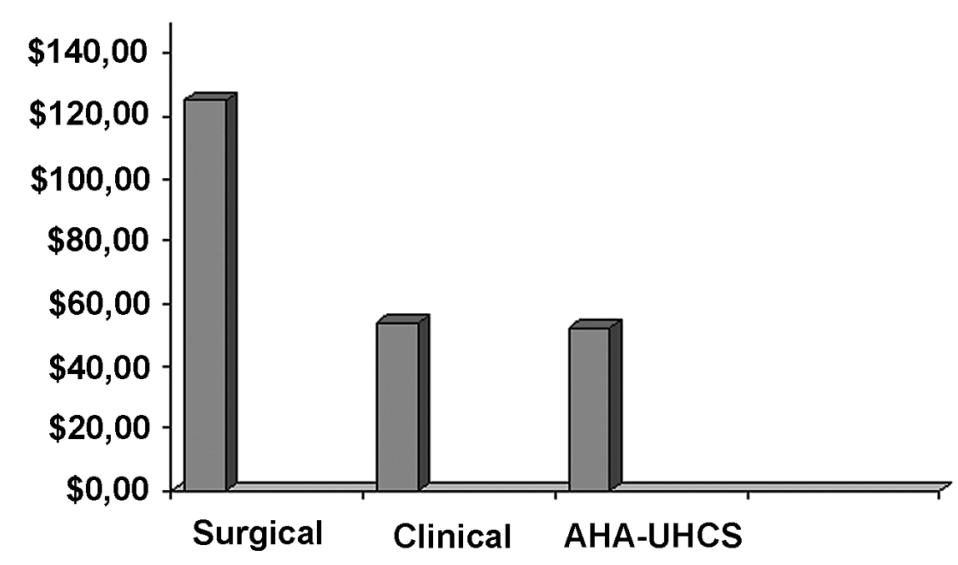

AHA-UHCS $=$ Authorized Hospital Admissions - Unified Health Care System

Figure 5-Comparison between the value transferred by the Unified Health Care System for the surgical procedure (circumcision) and median costs of the clinical and surgical groups. AHA-UHCS = Authorized Hospital Admissions-Unified Health Care System. 
There have been few studies comparing the economic impact of circumcision with that of topical treatment in individuals with phimosis. It has been estimated that topical treatment reduces costs by $75 \%$ in comparison with circumcision, representing, for example, a potential annual savings of 150 million francs in France $(17,18)$. However, in such studies, costs and treatment outcomes are estimated based on the means obtained in previous studies, and the potential economic risks of future complications are only speculated upon.

Van Howe (2004) conducted a cost-utility analysis of neonatal circumcision, evaluating the impact on the quality of life of patients for 72 years after circumcision (19). The author concluded that neonatal circumcision is more costly and has more adverse effects on the lifetime health of individuals than does not undergoing this procedure. Nevertheless, in a retrospective study evaluating the costs of circumcision, the authors concluded that neonatal circumcision provides medical benefit and prevents various pathologies, as well as costing ten times less than postnatal circumcision (20).

In general, pharmacoeconomic analysis consists of two essential elements: costs and outcome, which are, respectively, the nominator and the denominator of the equation. The pharmacoeconomic analysis in this study was based on cost minimization, in which we compared the costs of two treatment modalities whose final outcome measure was the resolution of pathologic phimosis (5).

The analysis showed that the costs of all items related to the clinical treatment were lower than were those related to the surgical treatment, except for the costs regarding medical visits and use of topical medication, which were not used in the surgical group patients. The evaluation showed that the clinical parameters, age brackets, follow-up rates and noncompliance rates were similar in the two groups. No differences were found between the groups in terms of surgical time or incidence of complications.

The cost savings corresponded to the total cost of treating the patients in the surgical group (US\$ $3,885.73$ ) subtracted from the total cost of treating the patients in the clinical group (US\$2,825.32). The cost savings, which was the amount of money saved by using the topical treatment as the first-line treatment, was US\$ 1,060.40.

Finally, when we compared the total costs of the treatment with the governmental reference values for circumcision, we found that amount provided by the health care system does not cover the costs of the surgical procedure. Therefore, topical corticosteroid administration as the first-line treatment for phimosis could represent a considerable savings to the institution.

We acknowledge some criticism of our study when considering that only complete exposure of the glans without a phimotic ring was defined as a favorable result of topic treatment. On the other hand, we tried to make the end-point of both kinds of treatment comparable and this could justify our methodology.

A second point of concern by some authors would be that our results with non-surgical treatment were inferior to most other published series that report higher (90-95\%) rates of efficiency. We stress that at the age we proposed to study these patients most boys had already experienced spontaneous resolution of phimosis, therefore, we were really comparing patients in the "waiting list" for surgery at our institution.

\section{CONCLUSIONS}

As a first-line treatment for phimosis, topical corticosteroid administration reduced costs by $27.3 \%$ in comparison with circumcision, even when we included the costs of the circumcisions eventually performed in the clinical treatment group. The mean cost of circumcision per child was US\$ 125.00 and for topical treatment was US\$ 54.00 , so that US\$ 35.00 could have been saved in the Brazilian public health system per child if conservative treatment had primarily been used.

These data underscore the concept that topical treatment should be offered to patients prior to considering surgery.

\section{ACKNOWLEDGEMENT}

Mrs. Rosana Lima Garcia Tsuji for valuable supported the pharmacoeconomic analysis. 


\section{CONFLICT OF INTEREST}

None declared.

\section{REFERENCES}

1. Agency for Healthcare Research and Quality. Most common surgical procedures in the United states 2003. Available at: http://www.ahcpr.gov. Accessed on April 11, 2008.

2. [No authors listed]: American Academy of Pediatrics: Report of the Task Force on Circumcision. Pediatrics. 1989; 84: 388-91. Erratum in: Pediatrics. 1989; 84: 761.

3. Kikiros CS, Beasley SW, Woodward AA: The response of phimosis to local steroid application. Ped Surg Int. 1993; 8: 329-32.

4. Jørgensen ET, Svensson A: The treatment of phimosis in boys, with a potent topical steroid (clobetasol propionate $0.05 \%$ ) cream. Acta Derm Venereol. 1993; 73: 55-6.

5. Reeder CE: Pharmacoeconomics and health care outcomes management: Focus on biotechnology. Am J Health Syst Pharm. 1995; 52 (Suppl 4): S5-8.

6. Kayaba H, Tamura H, Kitajima S, Fujiwara Y, Kato $\mathrm{T}$, Kato T: Analysis of shape and retractability of the prepuce in 603 Japanese boys. J Urol. 1996; 156: 18135.

7. Hutcheson JC: Male neonatal circumcision: indications, controversies and complications. Urol Clin North Am. 2004; 31: 461-7.

8. Schoen EJ, Wiswell TE, Moses S: New policy on circumcision--cause for concern. Pediatrics. 2000; 105: 620-3.

9. Cameron DW, Simonsen JN, D'Costa LJ, Ronald AR, Maitha GM, Gakinya MN, et al.: Female to male transmission of human immunodeficiency virus type 1: risk factors for seroconversion in men. Lancet. 1989; 2: 403-7.

10. Tran PT, Giacomantonio M: Routine neonatal circumcision? Can Fam Physician. 1996; 42: 2201-4.

11. Guerrero Fernández J, Guerrero Vázquez J, Russo de la Torre F: Conservative treatment of phimosis, balanitis and perianal streptococcal disease with local steroid therapy. An Pediatr (Barc). 2003; 58: 198-9.

12. Monsour MA, Rabinovitch HH, Dean GE: Medical management of phimosis in children: our experience with topical steroids. J Urol. 1999; 162: 1162-4.

13. DeVries CR, Miller AK, Packer MG: Reduction of paraphimosis with hyaluronidase. Urology. 1996; 48: 464-5.

14. Orsola A, Caffaratti J, Garat JM: Conservative treatment of phimosis in children using a topical steroid. Urology. 2000; 56: 307-10.

15. Chu CC, Chen KC, Diau GY: Topical steroid treatment of phimosis in boys. J Urol. 1999; 162: 861-3.

16. Garcia de Freitas R, Nobre YD, Demarchi GT, Hachul M, Macedo A Jr, Srougi M, et al.: Topical treatment for phimosis: Time span and other factors behind treatment effectiveness. J Pediatr Urol. 2006; 2: 380-5.

17. Van Howe RS: Cost-effective treatment of phimosis. Pediatrics. 1998; 102: E43.

18. Berdeu D, Sauze L, Ha-Vinh P, Blum-Boisgard C: Cost-effectiveness analysis of treatments for phimosis: a comparison of surgical and medicinal approaches and their economic effect. BJU Int. 2001; 87: 239-44.

19. Van Howe RS: A cost-utility analysis of neonatal circumcision. Med Decis Making. 2004; 24: 584601.

20. Schoen EJ, Colby CJ, To TT: Cost analysis of neonatal circumcision in a large health maintenance organization. J Urol. 2006; 175: 1111-5.

\section{Correspondence address:}

Dr. Yuri Dantas Nobre

Av. Senador César Vergueiro, 409

Ribeirão Preto, SP, 14020-500, Brazil

Fax: + 5516 3236-3646

E-mail: yuridantas@ig.com.br 


\section{EDITORIAL COMMENT}

This randomized trial compares the efficacy and costs of an 8 -week topical treatment with 0.2 betamethasone and hyaluronidase vs. primary circumcision in children with phimosis. The authors concluded that topical steroid treatment reduced the costs by $27.3 \%$ in comparison with circumcision. Only a few pharmacoeconomic analyses related to the treatment of these two groups have been published in the literature. However, I am skeptical about the definition of phimosis according to the Kayaba's (1) classification. What the authors defined as phimosis may well represent physiological phimosis. In the physiological phimosis, the non-retractile foreskin forms a normal and unscarred preputial orifice and when the foreskin is retracted, the preputial meatus opens as a flower. The appearance of pathological phimosis is clear with the preputial orifice white, indurated, and scarred. I am wondering whether children were treated because of physiological phimosis that would resolve spontaneously up to adolescence.
I must acknowledge the effort of the authors in setting this study together. However, there are some limitations related to this study. First, because of the relatively small number of patients the results of this trial may reflect a low statistical power rather than real differences between the two methods used. Second, we cannot exclude the possibility that another density of betamethasone would have different results than those observed here. How and why the authors chose a $0.2 \%$ betamethasone and hyaluronidase cream? An additional concern is that, although the authors compare a topic steroid to primary circumcision, one might question whether steroid is superior to a control group. Unless the authors include this group, the study may present biased and incomplete information.

\section{REFERENCE}

1. Kayaba H, Tamura H, Kitajima S, Fujiwara Y, Kato T, Kato T: Analysis of shape and retractability of the prepuce in 603 Japanese boys. J Urol. 1996; 156: 1813-5.

Dr. George Vaos

Department of Pediatric Surgery Alexandroupolis University Hospital Democritus University of Thrace School of Medicine Alexandroupolis, Greece E-mail:gvaos@hotmail.com

\section{EDITORIAL COMMENT}

Local corticosteroid application has become a popular alternative to the surgical treatment for phimosis during the last decade. Thus, it is useful to compare these treatment modalities also from the economical point of view. It was interesting that the nonsurgical treatment was more economical despite a quite high failure rate in this study. The reported success rates of the corticosteroid treatment are very variable, perhaps because of the patient selection. It is important to distinguish physiologic congenital 
phimosis and pathological phimosis with scars when deciding whether or not to treat. It has been shown that the incidence of phimosis is $50 \%$ at the age of 1 year, $8 \%$ at the age of 6 years and $1 \%$ at the age of 15 years (1). The possible health benefit for treating physiologic phimosis is controversial. In our hospital, physiologic phimosis is treated only in the rare cases with significant symptoms e.g. in voiding. In non-symptomatic cases, spontaneous resolution of phimosis is delayed until puberty. In pathologic phimosis, the prepuce is scarred mostly due to skin disease balanitis xerotica obliterans. Usually pathological phimosis is not observed before school age. In our hospital, the main indication for circumcision is pathological phimosis with scars. In the present series, no patient had pathological phimosis and few had symptoms. I find the indications for treatment unclear in this study and think that it could have been more economical not to treat most of these patients at all.

\section{REFERENCE}

1. Berdeu D, Sauze L, Ha-Vinh P, Blum-Boisgard C: Cost-effectiveness analysis of treatments for phimosis: a comparison of surgical and medicinal approaches and their economic effect. BJU Int. 2001; 87: 239-44.

Dr. Seppo Taskinen Department of Pediatric Surgery Helsinki University Central Hospital

Helsinki, Finland E-mail:seppo.taskinen@hus.fi 